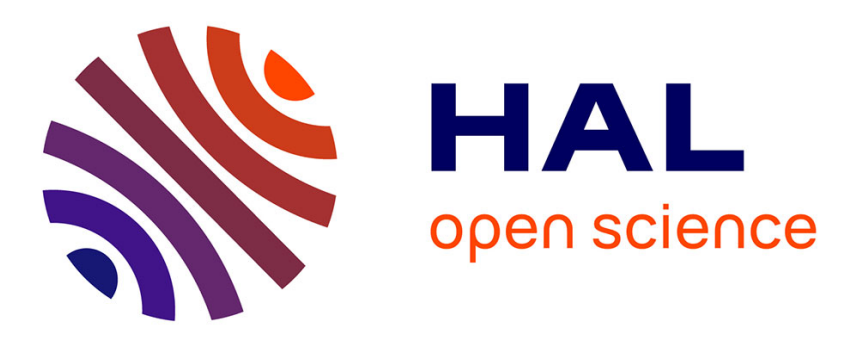

\title{
Kinematic analysis and dimensional optimization of a 2R2T parallel manipulator
}

\author{
Pedro Araujo-Gómez, Miguel A Díaz-Rodríguez, Vicente Mata, Octavio
} Andrés González Estrada

\section{- To cite this version:}

Pedro Araujo-Gómez, Miguel A Díaz-Rodríguez, Vicente Mata, Octavio Andrés González Estrada. Kinematic analysis and dimensional optimization of a $2 \mathrm{R} 2 \mathrm{~T}$ parallel manipulator. Journal of the Brazilian Society of Mechanical Sciences and Engineering, 2019, 41 (10), 10.1007/s40430-019-1934-1 . hal-02306621

\section{HAL Id: hal-02306621 \\ https://hal.science/hal-02306621}

Submitted on 6 Oct 2019

HAL is a multi-disciplinary open access archive for the deposit and dissemination of scientific research documents, whether they are published or not. The documents may come from teaching and research institutions in France or abroad, or from public or private research centers.
L'archive ouverte pluridisciplinaire HAL, est destinée au dépôt et à la diffusion de documents scientifiques de niveau recherche, publiés ou non, émanant des établissements d'enseignement et de recherche français ou étrangers, des laboratoires publics ou privés. 


\title{
Kinematic analysis and dimensional optimization of a $2 \mathrm{R} 2 \mathrm{~T}$ parallel manipulator
}

\author{
Pedro Araujo-Gómez ${ }^{1}$, Miguel Díaz-Rodríguez ${ }^{1}$, Vicente Mata $^{2}$, Octavio A. González-Estrada $^{3}$ \\ ${ }^{1}$ Laboratorio de Mecatrónica y Robótica MECABOT, Facultad de Ingeniería, Universidad de los Andes, Mérida, \\ 5101, Venezuela. \\ ${ }^{2}$ Centro de Investigación en Ingeniería Mecánica, Universitat Politècnica de València, Valencia, 46022, España, \\ Orcid: 0000-0003-2255-0567. \\ ${ }^{3}$ Energy and Environment Research Group - GIEMA, School of Mechanical Engineering, Universidad Industrial \\ de Santander, Bucaramanga, 680002, Colombia. Orcid: 0000-0002-2778-3389.
}

Correspondence author: Miguel Díaz-Rodríguez; dmiguel@ula.ve, tel: +584247019697.

\begin{abstract}
The need of a device providing two translational (2T) and two rotational (2R) movements led us to the design a 3UPS-1RPU parallel manipulator. The manipulator consisted on a mobile platform connected to a base through four legs. That is, the manipulator layout has one central leg and three external legs at the same radial distance. By studying different locations of the legs anchoring point, we improved the first layout design, yet not the optimal one. On this basis, this paper focus on the optimal dimensional design of the manipulator. To this end, we put forward the kinematics equations of the manipulator in accordance to the anchoring points coordinates. Through a numerical approach, the equations enable to find the manipulator workspace. Also, we find a global manipulability index using a local dexterity measure. The latter index serves as optimal function. The optimization process considers joint constraints. Thus, we built a nonlinear optimization problem solved through sequential quadratic programming algorithms. We start by optimizing only a small set of parameters rather than the entire set, which gives us insights on the initial guess to optimize using the entire set. The optimal design layout varies from the original layout. Findings suggest that a task-oriented reconfiguration strategy can improve manipulator performance.
\end{abstract}

\section{Keywords}

Parallel robots; optimization; kinematic analysis; dexterity.

\section{Introduction}

Bonev [1] demystifies that parallel manipulators (PMs) came after the serial manipulators. He suggested that the first industrial robot was already a parallel manipulator (PM) architecture. Pollard patented the robot cited in [1], which consisted of a 5 Degree of Freedom (DoF) PM developed as spray painting device, see [2]. However, most of the research papers in the field claim that the first PM architecture is the Gough's testing machine [3] or Stewart's flight simulator [4]. Since both 6-DoF architectures ([3] and [4]) appeared almost at the same time, the robot took the name of Gough-Stewart platform which consists of a mobile platform attached to a fixed base through 6 parallel legs. The kinematics [5], singularity [6], dynamics [7], modeling and control [8] of this PM have been a major topic of research in the past and it continues up until now, e.g. [9].

Some applications require less than 6-DoF. Let us take for example an ankle rehabilitation task. One of the first PM proposed for ankle rehabilitation was the Rutgers Ankle device, designed based on a 6-DoF architecture [10]. Nonetheless, ankle rehabilitation therapies require less than 6-DoF, references [11] and [12] have proposed PMs with 3-DoF, to name a few. PMs with fewer DOF can enter in the group of lower-mobility robots (LMR). These robots present a simpler mechanical design, lower manufacturing cost, and actuation cost compared to the 6-DoF platforms. Thus, strategies for developing lower-mobility PM for specific tasks demanding less than 6-DoF is relevant, and it is in the scope of this paper.

On the whole, PMs belong to one of the following categories: i) pure translational, ii) pure rotational, and iii) mixed mobility. The manipulators in the latter category convey both translational and rotational DoF. One of such manipulators is the two rotational and two translational (2R2T) architecture. References [14-17] presented contributions on designing 4-DoF PMs with 2R2T motion. Although, they focus on theoretical design without considering experimental prototyping or specific application. Instead, few works have considered a design for application approach. That is, for turbine blade machining in [18] and automated fiber placement in [19]. In the 
latter reference, authors found the layout of the manipulator through optimization. To our point of view, the literature dealing with 2R2T motion is scarce. Also, the manipulator presented so far, few of them have become actual prototypes.

Early works in the optimization of PM refer back to [20-21] where authors considered the optimum kinematic design of a planar PM. The paper introduced different criteria for establishing the optimal function for optimization, among the criteria they considered the global workspace and the isotropic condition of the manipulator. Other authors have considered the optimization approach to meet different criteria so they use multiobjective optimization algorithms [22]. Then, many works have considered the optimal design problem in PM. Most of the reviewed papers set the optimization process in order to obtain symmetric configuration, this means that the design of the legs is at the same radial distance for the fixed based and the platform. The idea is to develop a symmetric manipulator and to reduce the optimization parameters. Optimizing PM with 2T2R as such reduces the possibility to improve the manipulator performance. A new possibility for optimization we want to consider is to let the location of the anchoring points, the points connecting the legs to the platform, the variable in the optimization process.

Due to the need of a robot able to perform 2T2R movements at its end-effector, Araujo-Gómez et al. in [23] proposed a 3UPS-1RPU PM for knee rehagnosis (rehabilitation and diagnosis). Note that, U, P, R, and S stand for universal, prismatic, revolute and spherical joints. The manipulator consists of a 4 DoF PM where the mobile platform is connected to a base by means of four legs. The original layout of the manipulator has a central RPU leg and three external UPS legs. Reference [24] put forward the kinematic equations for the original layout. Also, reference [25] showed the mechatronics and the control architecture design of the prototype. On this basis, the paper aims to improve the design of the manipulator through optimization. Note that, Ref. [24] considers the anchoring points of the UPS legs at the same circle centered in the ends of the RPU leg. This approach reduces the possible arrangement of the manipulator layout. Instead, here the kinematics model considers each anchoring point at different radial distances, which gives more flexibility to find an improved layout. In addition, the optimization relies on a nonlinear optimization process considering a numerical computation of the workspace, the global condition index, and joints physical constraints.

Next section displays the equations for the kinematic analysis of the manipulator. Section 3 describes the numerical computation of the workspace, dexterity index, objective function and constraints use for the nonlinear optimization. Section 4 presents and discusses the results. Finally, Section 5 lists the conclusions and sheds some light on future works.

\section{Kinematic Analysis}

\subsection{The 3UPS-1RPS parallel manipulator}

Araujo-Gómez et al. in [23] developed the parallel manipulator that will be optimized in this paper. The manipulator's design considers that a rehagnosis task of the knee requires 2R2T motion to be applied on the foot of a lower limb. The manipulator consists of three outer legs and a central leg, see Figure 1. Each leg has two links, the proximal link near the base and the distal link attached to the platform. The external legs present a UPS architecture in which the anchoring points form a non-equilateral triangle both at the base and at the platform. The U-joints of the legs, connecting the base to the proximal link, have the same orientation which means that the first $Z_{i}$ axis of each $\mathrm{U}$-joint points toward the same direction. A P-joint connects the proximal link to the distal link, while an S-joint connects the distal link to the platform. The central leg of the manipulator has RPU architecture, an R-joint connects the base to the leg and a U-joint connects the leg to the platform. A rotational motor through a screw-ball system provides actuation on each P-joint. 


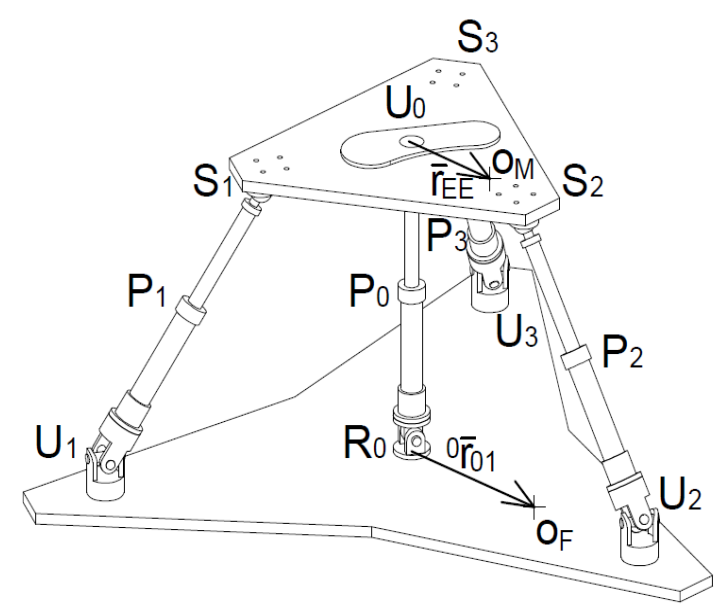

Figure 1: Schematic representation of the 3UPS-1RPS parallel manipulator as presented in [20].

\subsection{Inverse-displacement analysis}

The inverse-displacement equations developed in [24] consider the location of anchoring points of the UPS legs at the same radial distance, which means that the UPS legs are located in a circle of center at the ends of the RPU leg. The non-equilateral triangle shape is obtained by changing the angle of the radial direction defining the location of the leg. If we consider this case, only the radial distance (both for the base and the platform) and the angles will be fed to the optimization process, 6 design parameters will be the variables for the optimization, 2 distances, and 4 angles. Instead, we consider in this paper the location of the anchoring points as optimization variables. Thus, we require to present the inverse-displacement equations as a function of the anchoring-points coordinates.

Therefore, first, we establish the local coordinate system attached to the link on each robot leg through the Denavit-Hartenberg parameters according to Craig's notation [26], see Figure 2a for the UPS legs, and Figure 2b for the RPU leg. In the figure, subscripts F and M stand for the location of the coordinate system attached to the fixed base and the mobile platform, respectively, ${ }^{0} \vec{r}_{0 j}$ represents the position vector locating the origin of the $j$ leg with respect to the global coordinate system, ${ }^{M} \vec{r}_{E j}$ is the position vector locating the joint between the leg and the platform with respect to the local reference system attached to the platform. For the UPS leg, we define 6 local coordinate systems, while for the RPS only 4 coordinate systems are required. Tables 1 and 2 list the D-H parameters for the RPS leg and the UPS legs, where subscript $j i$ should be read as a coordinate system of the leg

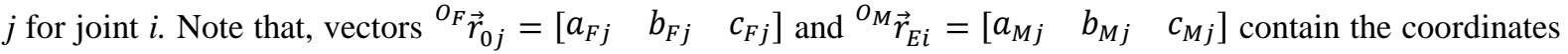
of the anchoring points, which are the variables for the kinematic analysis and the dimensional optimization.

In addition, we define the end-effector position and orientation using the position vector ${ }^{O_{F}} \vec{r}_{O_{F} O_{M}}=\left[\begin{array}{lll}X & Y & Z\end{array}\right]$ and the rotational matrix,

$$
\mathbf{R}_{T}=\mathbf{R} x \cdot \mathbf{R} y \cdot \mathbf{R} z=\left[\begin{array}{ccc}
c \gamma c \phi & -c \gamma s \phi & s \gamma \\
s \delta s \gamma c \phi+c \delta s \phi & -s \delta s \gamma s \phi+c \delta c \phi & -s \delta c \gamma \\
c \delta s \gamma c \phi+s \delta s \phi & c \delta s \gamma s \phi+s \delta c \phi & c \delta c \gamma
\end{array}\right]
$$

In equation (1), $\delta, \gamma, \phi$ are the Euler angles according to XYZ-fixed angle system. Also, $c *=\cos (*)$ and $s *=$ $\sin (*)$. 


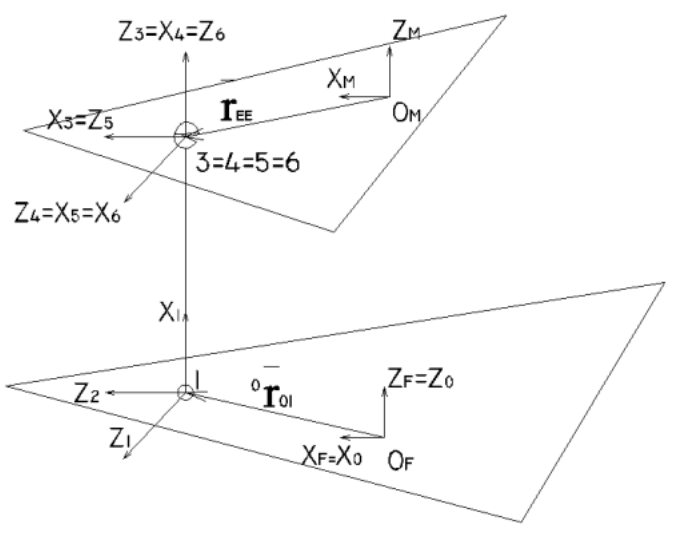

(a)

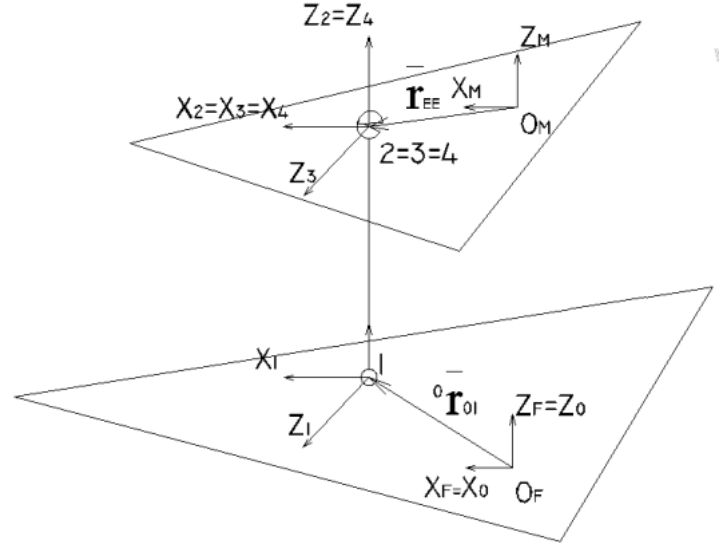

(b)

Figure 2: Location of the local coordinate systems. (a) UPS legs, (b) RPU leg.

Table 1: D-H parameters for the RPS.

\begin{tabular}{ccccc}
\hline$j i$ & $\alpha_{j, i-1}$ & $a_{j, i-1}$ & $d_{j, i}$ & $\theta_{j, i}$ \\
\hline 11 & $-90^{\circ}$ & 0 & 0 & $q_{1,1}$ \\
12 & $90^{\circ}$ & 0 & $q_{1,2}$ & 90 \\
13 & $-90^{\circ}$ & 0 & 0 & $q_{1,3}$ \\
14 & -90 & 0 & 0 & $q_{1,4}$ \\
\hline
\end{tabular}

Table 2: D-H parameters for UPS legs.

\begin{tabular}{ccccc}
\hline$j i$ & $\alpha_{j, i-1}$ & $a_{j, i-1}$ & $d_{j, i}$ & $\theta_{j, i}$ \\
\hline$i 1$ & $-90^{\circ}$ & 0 & 0 & $q_{i, 1}$ \\
$i 2$ & $90^{\circ}$ & 0 & 0 & $q_{i, 2}$ \\
$i 3$ & $0^{\circ}$ & 0 & $q_{i, 3}$ & 0 \\
$i 4$ & 90 & 0 & 0 & $q_{i, 4}$ \\
$i 5$ & $90^{\circ}$ & 0 & 0 & $q_{i, 5}$ \\
$i 6$ & $90^{\circ}$ & 0 & 0 & $q_{i, 6}$ \\
\hline
\end{tabular}

The inverse displacement consists of finding the actuation coordinates, having set the end-effector coordinates. The problem can be solved by using the vector-loop equations. For the RPU leg, a loop can be established between the position vector of the end-effector and the position of the RPU leg,

$$
{ }^{O_{F}} \vec{r}_{O_{F} O_{14}}={ }^{0} \vec{r}_{O_{F} O_{1}}+{ }^{0} \mathbf{R}_{11}{ }^{1} \vec{r}_{O_{1} O_{2}}=\left[\begin{array}{c}
a_{F 1}-s_{11} d_{1} \\
b_{F} \\
c_{F 1}+c_{11} d_{1}
\end{array}\right]
$$

and,

${ }^{o} \vec{r}_{O_{F} O_{4}}={ }^{o} \vec{r}_{O_{F} O_{M}}+\mathbf{R}_{T} \vec{r}_{O_{M} O_{14}}=\left[\begin{array}{c}X+c_{\gamma} c_{\phi} a_{M 1}-c_{\gamma} s_{\phi} b_{M 1}+s_{\gamma} c_{M 1} \\ Y+s_{\phi} a_{M 1}+c_{\phi} b_{M 1} \\ Z-s_{\gamma} c_{\phi} a_{M 1}+s_{\gamma} s_{\phi} b_{M 1}+c_{\gamma} c_{M 1}\end{array}\right]$

We can equate equations (2) and (3) to obtain the actuator displacement as, 
$d_{1}=\left[\left(X+c_{\gamma} c_{\phi} a_{M 1}-c_{\gamma} s_{\phi} b_{M 1}+s_{\gamma} c_{M i}-a_{F 1}\right)^{2}+\left(Z-s_{\gamma} c_{\phi} a_{M 1}+s_{\gamma} s_{\phi} b_{M 1}+c_{\gamma} c_{M 1}-c_{F 1}\right)^{2}\right]^{1 / 2}$

We can follow a similar approach to obtain the inverse-displacement equations for the UPS legs. That is, the loop equations are established based on the position vector of the end-effector and the position of each $j$ UPS leg for $j=2 . .4$ as follows,

$$
{ }^{o} \vec{r}_{O_{F} O_{j 6}}={ }^{o} \vec{r}_{O_{F} O_{i}}+{ }^{0} \mathbf{R}_{j 1}{ }^{1} \vec{r}_{O_{j 1} O_{j 2}}=\left[\begin{array}{c}
a_{F j}-c_{21} s_{22} d_{1} \\
b_{F j}-c_{22} d_{1} \\
c_{F j}+s_{21} s_{22} d_{1}
\end{array}\right]
$$

and,

$$
{ }^{o} \vec{r}_{O_{F} O_{j 6}}={ }^{o} \vec{r}_{O_{F} O_{M}}+R_{T} \vec{r}_{O_{M} O_{j 6}}=\left[\begin{array}{c}
X+c_{\gamma} c_{\phi} a_{M j}-c_{\gamma} s_{\phi} b_{M j}+s_{\gamma} c_{M j} \\
Y+s_{\phi} a_{M j}+c_{\phi} b_{M j} \\
Z-s_{\gamma} c_{\phi} a_{M j}+s_{\gamma} s_{\phi} b_{M j}+c_{\gamma} c_{M j}
\end{array}\right] .
$$

Similarly, we can equate equation (5) and (6) to obtain,

$$
\begin{gathered}
d_{j}=\left[\left(X+c_{\gamma} c_{\phi} a_{M i}-c_{\gamma} s_{\phi} b_{M i}+s_{\gamma} c_{M i}-a_{F i}\right)^{2}+\left(Y+s_{\phi} a_{M i}+c_{\phi} b_{M i}-b_{F i}\right)^{2}++\left(Z-s_{\gamma} c_{\phi} a_{M i}+\right.\right. \\
\left.\left.\quad s_{\gamma} s_{\phi} b_{M i}+c_{\gamma} c_{M i}-c_{F i}\right)^{2}\right]^{1 / 2}
\end{gathered}
$$

Summarizing, the leg displacement $d_{j}$ from $j=1 . .4$ can be found using equations (4) and (7). The actuator displacement will be a function of 23 parameters. As a design consideration, we constrain the location of the 4 joints of the platform to the same plane. Similarly, the location of base joints shares the same plane. In this way, the optimization process considers 15 parameters.

\subsection{Dexterity analysis}

First, we have to define the linear and angular velocity of the platform. Then, the speed of the actuators can be found given the velocity of the end-effector. In this sense, the linear velocity is given by the velocity of the local coordinate system $O_{M}, V_{O_{M}}=\left[\dot{X}_{M} \dot{\mathrm{Y}}_{M} \mathrm{Z}_{M}\right]^{T}$, while the angular velocity is obtained from the partial derivatives of the rotational matrix, equation (1). That is,

$$
{ }^{o} \omega_{M}=\left[\begin{array}{c}
\dot{\delta} \\
0 \\
0
\end{array}\right]+\mathbf{R}_{X}\left[\begin{array}{l}
0 \\
\dot{\gamma} \\
0
\end{array}\right]+\mathbf{R}_{X} \cdot \mathbf{R}_{Y}\left[\begin{array}{l}
0 \\
0 \\
\dot{\phi}
\end{array}\right]=\left[\begin{array}{c}
s_{\gamma} \dot{\phi} \\
\dot{\gamma} \\
c_{\gamma} \dot{\phi}
\end{array}\right]
$$

Due to the fact that rotation in the $\mathrm{X}$ direction is zero, we have $\dot{\delta}=0$.

The linear velocity of the U-joint connecting the RPU leg to the platform can be found by differentiation of ${ }^{F} \vec{r}_{F O_{14}}$ on equation (2),

$$
\frac{\delta^{F} \vec{r}_{F O_{14}}}{\partial q_{j i}}=\left[\begin{array}{c}
-c_{11} d_{1} \dot{\theta}_{11}-s_{11} \dot{d}_{1} \\
0 \\
-s_{11} d_{1} \dot{\theta}_{11}+c_{11} \dot{d}_{1}
\end{array}\right]
$$

The same velocity can be obtained through the end-effector velocity as follows, 


$$
F \vec{r}_{F O_{14}}=\left[\begin{array}{c}
\dot{X}_{M}-\left(s_{\gamma} c_{\phi} a_{M 1}+s_{\gamma} s_{\phi} b_{M 1} c_{\gamma} c_{M 1}\right) \dot{\gamma}-c_{\gamma}\left(s_{\phi} a_{M 1}+c_{\phi} b_{M 1}\right) \dot{\phi} \\
\dot{Y}_{M}+\left(c_{\phi} a_{M 1}-s_{\phi} b_{M 1}\right) \dot{\phi} \\
\dot{Z}_{M}-\left(c_{\gamma} c_{\phi} a_{M 1}-c_{\gamma} s_{\phi} b_{M 1}+s_{\gamma} c_{M 1}\right) \dot{\gamma}+s_{\gamma}\left(s_{\phi} a_{1 M}+c_{\phi} b_{M 1}\right) \dot{\phi}
\end{array}\right] .
$$

The equation for the velocity of the actuator is found by i) equating equations (9) and (10), ii) multiplying the first row by $s_{11}$, ii) multiplying the third row by $c_{11}$, and iv) adding the first and third rows. The resulting equation gives the relationship between the actuator and end-effector velocity. That is,

$$
\begin{aligned}
& \dot{d}_{1}=-s_{11} \dot{X}_{M}+c_{11} \dot{\mathrm{Z}}_{M}+\left(s_{11}\left(s_{\gamma} c_{\phi} a_{M 1}+s_{\gamma} s_{\phi} b_{M 1} c_{\gamma} c_{M 1}\right)-c_{11}\left(c_{\gamma} c_{\phi} a_{M 1}-c_{\gamma} s_{\phi} b_{M 1}+s_{\gamma} c_{M 1}\right)\right) \dot{\gamma}+ \\
& \left(s_{11} c_{\gamma}\left(s_{\phi} a_{M 1}+c_{\phi} b_{M 1}\right)+c_{11} s_{\gamma}\left(s_{\phi} a_{M 1}+c_{\phi} b_{M 1}\right)\right) \dot{\phi}
\end{aligned}
$$

The second row of the velocity equations introduces the following constraint,

$$
\dot{Y}_{M}+\left(c_{\phi} a_{M 1}-s_{\phi} b_{M 1}\right) \dot{\phi}=0 .
$$

We follow a similar approach for the UPS legs. First, equation (5) is differentiated,

$$
\frac{\delta^{{ }^{0} \vec{r}_{F O}}}{\partial q_{j i}}=\left[\begin{array}{c}
s_{21} s_{22} d_{1} \dot{\theta}_{21}-c_{21} c_{22} d_{1} \dot{\theta}_{22}-c_{21} s_{22} \dot{d}_{1} \\
s_{22} d_{1} \dot{\theta}_{22}-c_{22} \dot{d}_{1} \\
c_{21} s_{22} d_{1} \dot{\theta}_{21}+s_{21} c_{22} d_{1} \dot{\theta}_{22}+s_{21} s_{22} \dot{d}_{1}
\end{array}\right] .
$$

Then, we found the velocity of $O_{j 6}$ through the velocity of the end-effector. That is,

$$
F \vec{r}_{F O_{j 6}}=\left[\begin{array}{c}
\dot{\mathrm{X}}_{M}-\left(s_{\gamma} c_{\phi} a_{M j}+s_{\gamma} s_{\phi} b_{M j}+c_{\gamma} c_{M j}\right) \dot{\gamma}-c_{\gamma}\left(s_{\phi} a_{M j}+c_{\phi} b_{M j}\right) \dot{\phi} \\
\dot{\mathrm{Y}}_{M}+\left(c_{\phi} a_{M j}-s_{\phi} b_{M j}\right) \dot{\phi} \\
\dot{Z}_{M}-\left(c_{\gamma} c_{\phi} a_{M j}-c_{\gamma} s_{\phi} b_{M j}+s_{\gamma} c_{M j}\right) \dot{\gamma}+s_{\gamma}\left(s_{\phi} a_{M j}+c_{\phi} b_{M j}\right) \dot{\phi}
\end{array}\right] .
$$

The equation for the velocity of the actuators for $j=2 . .4$ are found by i) equating equations (13) and (14), ii) multiplying the first row by $-c_{j 1} s_{j 2}, 3$ ) multiplying the second by row $-c_{j 2}$, iii) multiplying the third row by $s_{j 1} s_{j 2}$, and iv) adding the three rows. The resulting equation gives the relationship between the actuator and endeffector velocity. That is,

$\dot{d}_{j}=-c_{j 1} s_{j 2} \dot{X}_{M}+s_{j 1} s_{j 2} \dot{Z}_{M}+\left(c_{j 1} s_{j 2}-s_{j 1} s_{j 2}\left(c_{\gamma} c_{\phi} a_{M j}-c_{\gamma} s_{\phi} b_{M j}+s_{\gamma} c_{M j}\right)\right) \dot{\gamma}+\left(c_{j 1} s_{j 2} c_{\gamma}\left(s_{\phi} a_{M j}+\right.\right.$

$\left.\left.c_{\phi} b_{M j}\right)+c_{j 2}\left(-c_{\phi} a_{M j}+s_{\phi} b_{M j}\right)+s_{j 1} s_{j 2} s_{\gamma}\left(s_{\phi} a_{M j}+c_{\phi} b_{M j}\right)\right) \dot{\phi}$.

Equations (11) and (15) establishing the relationship between the speed of the actuators and the end-effector velocity can be written in matrix form as,

$$
\dot{d}_{j}=\mathrm{J} \dot{x} .
$$

In equation (16), $\dot{x}=[\dot{X}, \dot{\mathrm{Z}}, \dot{\gamma}, \dot{\phi}]^{T}, \dot{d}_{j}=\left[\dot{d}_{1}, \dot{d}_{2}, \dot{d}_{3}, \dot{d}_{4}\right]^{T}, \mathrm{~J}$ is the Jacobian matrix. 
In equation (16) the Jacobian matrix produces a linear transformation between $\dot{x}$ and $\dot{d}_{j}$; however, the end-effector coordinates present two rotations and two translations. Thus, the transformation is not dimensionally homogenous. To avoid this unit incongruence, we divide the third and four columns of matrix $\mathbf{J}$ by the radius of the original platform design [27].

\subsection{Passive joints displacement.}

The kinematic equations are function of the passive joints. Computing the equations requires the angles of rotation of the R joint and the $\mathrm{U}$ joints. Starting from equation (3), we can isolate the $S_{11}$ and $C_{11}$ from the first and third row. Then,

$$
\theta_{11}=\arctan \left(\frac{-X-c_{\gamma} c_{\phi} a_{M 1}+c_{\gamma} s_{\phi} b_{M 1}-s_{\gamma} c_{M 1}+a_{F 1}}{Z-s_{\gamma} c_{\phi} a_{M 1}+s_{\gamma} s_{\phi} b_{M 1}+c_{\gamma} c_{M}-c_{F 1}}\right)
$$

Similarly, for the UPS leg,

$$
\theta_{j 1}=\arctan \left(\frac{Z-s_{\gamma} c_{\phi} a_{M j}+s_{\gamma} s_{\phi} b_{M j}+c_{\gamma} c_{M j}-c_{F j}}{-X-c_{\gamma} c_{\phi} a_{M j}+c_{\gamma} s_{\phi} b_{M j}-s_{\gamma} c_{M j}+a_{F j}}\right)
$$

The angle $\theta_{j 2}$ can be obtained by multiplyting the third row of equation (5) and (6) by $s_{j 1}$, and then, the resulting equation is subtracted by the first row previously multiplied by $c_{j 1}$. After some manipulation, we can get,

$$
\theta_{j 2}=\arctan \left(\frac{s_{21}\left(Z-s_{\gamma} c_{\phi} a_{M j}+s_{\gamma} s_{\phi} b_{M j}+c_{\gamma} c_{M j}-c_{F 1}\right)-c_{21}\left(X+c_{\gamma} c_{\phi} a_{M j}-c_{\gamma} s_{\phi} b_{M j}+s_{\gamma} c_{M j}-a_{F j}\right)}{-Y-s_{\phi} a_{M j}-c_{\phi} b_{M j}+a_{F j}}\right)
$$

\section{Workspace and Manipulability Index}

We estimate the workspace of the PM using a numerical approximation similar to the approach used in Ref. [28]. First, we define a given box with a size greater than the estimated size of the PM. On this preliminary box, we perform a point-to-point evaluation of all the kinematics equations. That is, at each point we determine whether the length of legs can be reached by actuators. If they are within range $(60$ and $90 \mathrm{~cm}))$ the point is considered feasibly otherwise it is dismissed. Each point represents a differential box, by adding all the boxes we can find the value of the workspace. Note that, the more feasibly points we have, the bigger the size of the workspace will be.

In addition, for each point, we compute the determinant of the Jacobian matrix (with homogenous dimensions) as follows,

$\operatorname{Det}\left(\mathrm{J}\left(a_{M j}, b_{M j}, c_{M j}, a_{F j}, b_{F j}\right)\right)<0.01$

We considered 0.01 as a threshold defining whether the point is dexterous or not. Moreover, at each point, the condition number is evaluated in order to determine the overall condition index. We have considered the average of all points using Yoshikawa manipulability index [29],

$I G C=\frac{1}{n} \sum_{i=1}^{n}\left(\operatorname{Det}\left(\mathbf{J}\left(a_{M j}, b_{M j}, c_{M j}, a_{F j}, b_{F j}\right)\right)\right)^{-1 / 2}$

Equation (21) defines the objective function of the optimization problem, the nonlinear constraints are given by joints physical constraints.

\section{Results and Discussion}

First row on Table 3 lists the result of evaluating the IGC for the original layout of the 3-UPS-1-RPS PM, see also Figure $3 \mathrm{a}$. In the table, the first column presents the results of the IGC computed using equation (21). In addition, we evaluate the number of points that can be reached by the manipulator $\left(N^{o} W S\right)$ which are listed in the second column. Also the number of non-dexterous points $\left(N^{o} N D\right)$, third column. Finally, for comparison purpose, the fourth column lists the percentage of non-dexterous points with respect to the points belonging to the workspace. 
Second row shows the results for a layout obtained through an optimization that considers as variables the position of anchoring points for the RPU leg. The remaining anchoring points are the same as the original layout. The optimization considers four variables $\left(a_{M 1}, b_{M 1}, a_{F 1}, b_{F 1}\right)$, which are the coordinates $(x, y)$ of the R-joint and the $\mathrm{U}$-joint respectively. The objective function for the optimization is given by $I G C$. We take advantages of the Matlab built-in routine fmincon for solving the nonlinear constraint optimization problem. We consider the coordinates $(x, y)$ of the original layout as an initial guess. We can see on the Table 3 that while the IGC is lower than that of the original layout, the percentages of non-dexterous points are greater, thus the obtained configuration cannot be considered as an improved design.

Third, fourth and fifth rows provide the results when optimizing the position of the first, second and third UPS legs, respectively while the other legs remain fixed. For each case, the initial guess corresponds to the PM original layout. From the table, we can see that when optimizing first and second leg the IGC reduces; however, the ratio non-dexterous/feasible points is higher than the original layout. The worst case is given when optimizing the third leg.

One of the main problem when optimizing is how to set up the initial guess. The above cases the initial guess considers the original layout. In this paper, we follow a cascade process of using the initial guess the results optimizing the legs each one at that time. That is, we optimize the first UPS anchoring points while the other UPS anchoring points equal to the original layout but the points of the RPS leg are given by the anchoring points found in the second optimization -results from the second row on Table 3. In this case, the IGC reduces to almost half of the original layout. Conversely, the ND/WS percentage is twice higher. Still, we cannot consider this case as an improvement with respect to the original design.

Table 3: Results for the optimization cases.

\begin{tabular}{lcccc}
\hline & $I G C$ & $N^{o} W S$ & $N^{o} N D$ & $N D / W S(\%)$ \\
\hline Original layout & 6,0393 & 1780 & 130 & $7,30 \%$ \\
Optimization RPU leg & 5,1903 & 1793 & 511 & $28,50 \%$ \\
Optimization UPS 1-leg & 4,1590 & 3287 & 613 & $18,65 \%$ \\
Optimization UPS 2-leg & 4,4537 & 2076 & 520 & $25,05 \%$ \\
Optimization UPS 3-leg & 5,3254 & 1780 & 566 & $31,80 \%$ \\
Optimization UPS 1-leg* & 3,8288 & 2163 & 350 & $16,18 \%$ \\
Optimization UPS 2-leg* & 3,1992 & 2365 & 217 & $9,18 \%$ \\
Optimization UPS 3-leg* & 3,1072 & 1641 & 76 & $4,63 \%$ \\
Optimization all legs* & $\mathbf{2 , 3 8 2 9}$ & $\mathbf{1 5 9 8}$ & $\mathbf{5 1}$ & $\mathbf{3 , 1 9 \%}$ \\
Optimization all legs** & $\mathbf{3 , 0 4 7 9}$ & $\mathbf{1 6 9 0}$ & $\mathbf{6}$ & $\mathbf{0 , 3 6 \%}$ \\
\hline
\end{tabular}

*Optimization considers as anchoring points of the remaining legs values from previous optimizations

Following the proposed cascade process, we optimize the $I G C$ considering as variables the anchoring points of the second UPS leg. In this case, the anchoring points of the central leg are the one obtained for the second row of Table 3, and the anchoring points of the first leg correspond to those obtained in the previous optimization. In this case, we have found a slight improvement of the IGC compare to the last case, but more importantly, the ND/WS percentage is close to the one of the original design. The result suggests us to further continue with the cascade process. Rows seven and eight present results when optimizing a single leg considering the anchoring points of the fixed legs from previous optimization results. By doing so, we were able to reduce both the $I G C$ and the $N D / W S(\%)$.

The layout found by optimizing the anchoring points of each leg one at the time, and also considering results from previous optimization allowed us to improve the original design. However, to search for further improvements, we consider all the anchoring points as parameters for the optimization. The initial guess was the one found in the latter case. In Table 3 we can see that the obtained solution represents an improvement when considering both the $I G C$ and the ND/WS (\%). Last row presents the result from an optimization similar to row eight but in this case, the objective function is given by the percentage of dexterous points. As can be seen in the table, the optimization provides a solution with lower $N D / W S(\%)$, yet with slightly higher values of $I G C$. This suggests that both indexes at some point are conflicting, thus a trade-off between both solution (eight and nine rows) can lead to an improved design. 
Figure $3 b$ presents a sketch of the optimal layout found in the latter optimization. The improvement on the kinematic performance is achieved by a configuration in which the central leg points toward the first leg. Finally, Figure 4 presents the prototype with the optimized layout.

$\mathrm{U} 2$

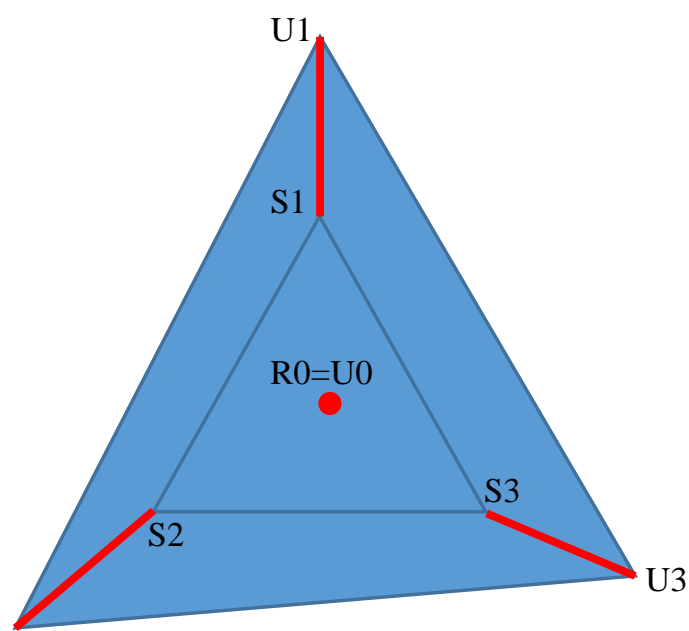

(a)

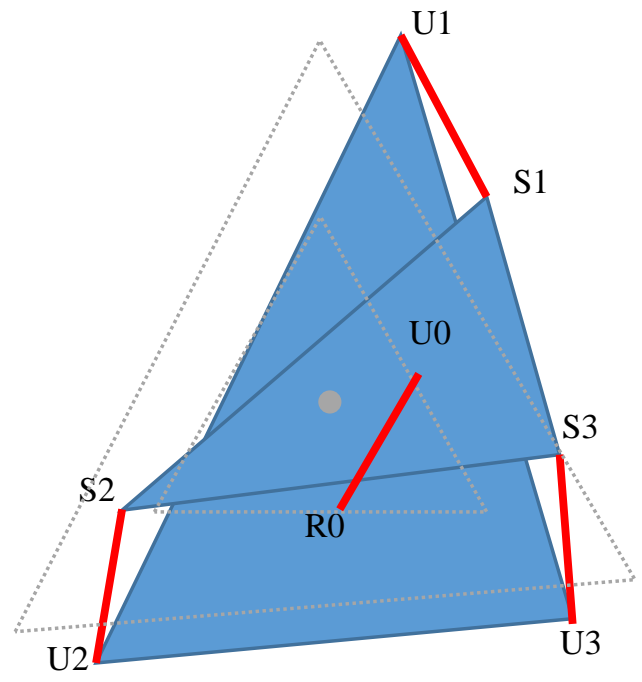

(b)

Figure 3: Representations PM layout. (a) Original layout as in [20], (b) optimal layout found in this paper.

As can be seen in Table 3, the final layout still has some non-dexterous configurations within its workspace. This fact represents one of the main drawbacks of PMs. Strategies for avoiding such configurations have been a topic of great interest, e.g., methods of passing through the configuration using actuation redundancy or another similar method like the one presented in [30] or [31], to name a few. However, we recommend that for a specific task it would be better to follow a reconfigurable strategy. Therefore, the steps ahead in our future work will start with the result found in this paper, and then we will focus on the design of a reconfigurable parallel manipulator with 2R2T motion.

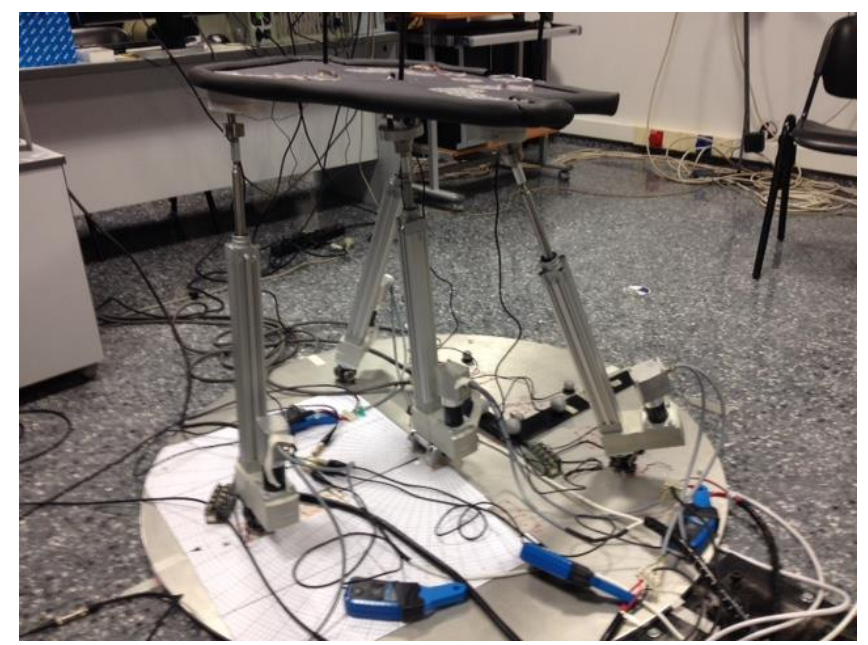

Figure 4: Prototype of the 3-UPS-1RPS PM at the Universitat Politècnica de València, Spain.

\section{Conclusions}

Starting from a previously proposed design and following a sequential optimization approach, in this paper, we have improved the design of a 4 DoF 3UPS-1RPS parallel manipulator. The manipulator has the characteristic that can tackle applications in which two rotational and two translational motion at the end-effector are required (2R2T). Note that in the field of parallel manipulators much of the work done is focused on 6-DoF, 4 DoF SCARA 
motion (3T1R), 3 DoF (3T, 2R1T, or 3R) and $2 \mathrm{DoF}$, few papers have dealt with the optimal design of 2R2T manipulator. This paper has presented the steps we have followed in order to optimize a manipulator of such kind. In this sense, we have solved a nonlinear optimization process including joints constraints. Most of the effort conducted in this work was performed in order to establish an initial guess to feed the optimization process. The objective function considers global manipulability condition index and also the percentage of non-dexterous points within its workspace. The approach presented in this paper can be used as guideline or a follow through steps for other works aim at customizing the design PMs with similar architecture. From our experience, the design of manipulator with 2R2T movements without singularities or non-dexterous points within its workspace is an intricate task. If more dexterous capabilities for specifics tasks are required, we recommend for further works to consider a reconfigurable strategy which is a brand new field currently developing in the parallel manipulator community.

\section{Acknowledgments}

This work was partially supported by the Spanish Ministry of Education, Culture, and Sports through the research project [DPI2013-44227-R]. We want to thank Professor Angel Valera and his team at the Laboratorio de Robótica, Universitat Politècnica de València for the image of the optimized manipulator's layout.

\section{References}

[1] I. Bonev, The true origins of parallel robots. ParalleMIC, https://www.parallemic.org/ 2003.

[2] Pollard, W. L. (1942). U.S. Patent No. 2,286,571. Washington, DC: U.S. Patent and Trademark Office.

[3] V. E. Gough and S. G. Whitehall, "Universal Tyre Test Machine”. Proceedings of 9th International Congress FISITA, May 1962, pp. 117-137, 1962.

[4] D. Stewart, “A Platform with Six Degrees of Freedom”. Proceedings of the Institution of Mechanical Engineers, vol. 180, no. 1, pp. 371-386, 1965.

[5] K. Harib and K. Srinivasan, "Kinematic and dynamic analysis of Stewart platform-based machine tool structures”. Robotica, vol. 21, no. 5, pp. 541-554, 2003.

[6] B.M. St-Onge and C.M. Gosselin, "Singularity analysis and representation of the general Gough-Stewart platform”. The International Journal of Robotics Research, vol. 19, no. 3, pp. 271-288, 2000.

[7] M.J. Liu, C.X. Li and C.N. Li, "Dynamics analysis of the Gough-Stewart platform manipulator". IEEE Transactions on robotics and automation, vol. 16, no. 1, pp- 94-98, 2000.

[8] Y. Ting, Y.S. Chen and H.C. Jar, "Modeling and control for a Gough- Stewart platform CNC machine". Journal of Field Robotics, vol. 21, no. 11, pp. 609-623, 2004.

[9] F. Wei, S. Wei, Y. Zhang and Q. Liao, "Forward Displacement Analysis of a General 6-3 Stewart Platform Using Conformal Geometric Algebra”. Mathematical Problems in Engineering, vol. 2017, Article ID 4687638, 9 pages, 2017.

[10] M. Girone, G. Burdea, M. Bouzit, V. Popescu and J.E. Deutsch, A Stewart platform-based system for ankle telerehabilitation. Autonomous robots, vol. 10, no. 2, pp. 203-212, 2001.

[11] C. Wang, Y. Fang, S. Guo and Y. Chen, "Design and kinematical performance analysis of a 3-RUS/RRR redundantly actuated parallel mechanism for ankle rehabilitation”. Journal of Mechanisms and Robotics, vol. 5, no. 4, pp. 041003, 2013.

[12] M. Vallés, J. Cazalilla, A. Valera, V. Mata, A. Page and M. Díaz-Rodríguez, "A 3-PRS parallel manipulator for ankle rehabilitation: towards a low-cost robotic rehabilitation”. Robotica, vol. 35, no. 10, pp. 1939-1957, 2017. 
[13] Y. Qiang, L. Bin, L. Yangmin and Z. Xinhua, "Kinematics Comparative Study of Two Overconstrained Parallel Manipulators," Mathematical Problems in Engineering, vol. 2016, Article ID 5091405, 12 pages, 2016.

[14] W. J. Chen, M.Y. Zhao, J.P. Zhou and Y.F. Qin, “A 2T-2R, 4-DoF parallel manipulator”. In ASME 2002 International Design Engineering Technical Conferences and Computers and Information in Engineering Conference ASME, pp. 881-885, 2002.

[15] C. Fan, H. Liu, G. Yuan and Y. Zhang, "A novel 2T2R 4-DOF parallel manipulator". In Knowledge Acquisition and Modeling (KAM), 2011 Fourth International Symposium on IEEE, pp. 5-8, 2011.

[16] C. Fan, H. Liu and Y. Zhang, "Type synthesis of 2T2R, 1T2R and 2R parallel mechanisms". Mechanism and Machine Theory, vol. 61, pp. 184-190, 2013.

[17] F. Xie, T. Li and X. Liu, "Type synthesis of 4-DOF parallel kinematic mechanisms based on Grassmann line geometry and atlas method". Chinese Journal of Mechanical Engineering, vol. 26, no. 6, pp. 10731081, 2013.

[18] H. Ghaffari, G. Payeganeh and M. Arbabtafti, "Kinematic design of a novel 4-DOF parallel mechanism for turbine blade machining". The International Journal of Advanced Manufacturing Technology, vol. 74, no. 5-8, pp. 729-739, 2014.

[19] D. Gan, J.S. Dai, J. Dias, R. Umer and L. Seneviratne, "Singularity-free workspace aimed optimal design of a 2T2R parallel mechanism for automated fiber placement". ASME Journal of Mechanisms and Robotics, vol. 7, no. 4, pp. 041022, 2015.

[20] C. M. Gosselin and J. Angeles. "The optimum kinematic design of a planar three-degree-of freedom parallel manipulator". ASME Journal of Mechanisms, Transmission and Automation in Design, vol. no. 110 pp. 35-41, 1988.

[21] C. M. Gosselin and J. Angeles. "The optimum kinematic design of a spherical three-degree-of freedom parallel manipulator". ASME Journal of Mechanisms, Transmissions and Automation in Design, vol. 111 no. 2, pp. 202-207, 1989.

[22] F. Hao and J.-P. Merlet. "Multi-criteria optimal design of parallel manipulators based on interval analysis". Mechanism and Machine Theory, vol. 40 no. 2 pp:157-171, 2005.

[23] P. Araujo-Gómez, M. Díaz-Rodríguez, V. Mata, A. Valera and A. Page, "Design of a 3-UPS-RPU parallel robot for knee diagnosis and rehabilitation”, In ROMANSY 21-robot design, dynamics and control, pp. 303-310, 2016.

[24] P. Araujo-Gómez, V. Mata, M. Díaz-Rodríguez, A. Valera and A. Page, "Design and Kinematic Analysis of a Novel 3UPS/RPU Parallel Kinematic Mechanism with 2T2R Motion for Knee Diagnosis and Rehabilitation Tasks". Journal of Mechanisms and Robotics, vol. 9, no. 6, pp. 061004, 2017.

[25] M. Vallés, P. Araujo-Gómez, V. Mata et al., "Mechatronic design, experimental setup, and control architecture design of a novel 4 DoF parallel manipulator". Mechanics Based Design of Structures and Machines, pp. 1-15, 2018.

[26] J.J. Craig, Introduction to robotics: mechanics and control, Pearson/Prentice Hall, New York, USA, 2005.

[27] J. Angeles, C. S. López-Cajún, "Kinematic isotropy and the conditioning index of serial robotic manipulators". The International Journal of Robotics Research, vol. 11, no. 6, pp. 560-571, 1992.

[28] A. Ramirez-Matheus, M. Díaz-Rodríguez and O.A. González-Estrada, "Estrategia de optimización para la síntesis dimensional de un robot paralelo 5R para una aplicación de mesa de corte / An approach for 
optimal dimensional synthesis of a 5R parallel robot for a CNC X-Y cutter”. Revista UIS Ingenierías, vol. 16, no. 2, pp. 197-206, 2017.

[29] T. Yoshikawa, "Manipulability of robotic mechanisms". The International Journal of Robotics Research, vol. 4, no 2, pp. 3-9, 1985.

[30] S.S. Parsa, R. Boudreau and J.A. Carretero, "Reconfigurable mass parameters to cross direct kinematic singularities in parallel manipulators". Mechanism and Machine Theory, vol. 85, pp. 53-63, 2015.

[31] M. Özdemir, "Dynamic analysis of planar parallel robots considering singularities and different payloads". Robotics and Computer-Integrated Manufacturing, vol. 46, pp. 114-121, 2017. 